\title{
Neutron Irradiation Induced Microstructural Changes in NBG-18 and IG-110 Nuclear Graphites
}

\author{
Chinnathambi Karthik ${ }^{1,2, *}$, Joshua Kane ${ }^{1,2,3}$, Darryl P. Butt ${ }^{1,2}$, William E. Windes ${ }^{2,3}$ and Rick Ubic ${ }^{1,2}$ \\ ${ }^{1}$ Department of Materials Science and Engineering, Boise State University, 1910 University Drive, \\ Boise, ID 83725. \\ ${ }^{2}$ Center for Advanced Energy Studies, 995 University Blvd, Idaho Falls, ID 83415. \\ ${ }^{3}$ Idaho National Laboratory, 2351 N. Boulevard, Idaho Falls, ID 83415.
}

\begin{abstract}
This paper reports the neutron-irradiation-induced effects on the microstructure of NBG-18 and IG-110 nuclear graphites. The high-temperature neutron irradiation at two different irradiation conditions was carried out at the Advanced Test Reactor National User Facility at the Idaho National Laboratory. NBG-18 samples were irradiated to $1.54 \mathrm{dpa}$ and $6.78 \mathrm{dpa}$ at $430{ }^{\circ} \mathrm{C}$ and $678{ }^{\circ} \mathrm{C}$ respectively. IG-110 samples were irradiated to $1.91 \mathrm{dpa}$ and $6.70 \mathrm{dpa}$ at $451^{\circ} \mathrm{C}$ and 674 ${ }^{\circ} \mathrm{C}$ respectively. Bright-field transmission electron microscopy imaging was used to study the changes in different microstructural components such as filler particles, microcracks, binder and quinoline-insoluble (QI) particles. Significant changes have been observed in samples irradiated to about $6.7 \mathrm{dpa}$. The closing of pre-existing microcracks was observed in both the filler and the binder phases. The binder phase exhibited substantial densification with near complete elimination of the microcracks. The QI particles embedded in the binder phase exhibited a complete microstructural transformation from rosettes to highly crystalline solid spheres. The lattice images indicate the formation of edge dislocations as well as extended line defects bridging the adjacent basal planes. The positive climb of these dislocations has been identified as the main contributor to the irradiation-induced swelling of the graphite lattice.
\end{abstract}

*Corresponding author. Tel: +1 208426 4646. E-mail: karthikchinnathambi@boisestate.edu, 


\section{Introduction}

Nuclear graphite has been used in reactors starting from the early graphite piles to fully commercialized high-temperature gas-cooled reactors (HTGRs) of the 1970s and 80s. Nuclear graphite will also be used in the next-generation very-high-temperature gas-cooled reactor (VHTR) as a structural material and moderator due to its superior neutron-moderation properties, high-temperature thermal and mechanical stability, easy machinability and cost-effectiveness [1, 2]. Although polycrystalline graphite has been used in nuclear applications since the first critical reactor, there is a lack of complete understanding of the properties mainly due to its very complex microstructure and difficulties associated with neutron-irradiation experiments.

The response of nuclear graphite to neutron irradiation may be considered to have two components, viz., intrinsic lattice effects and bulk microstructural effects. Under irradiation, graphite single crystals undergo lattice expansion along the c-axis and shrinkage parallel to the basal planes $[2,3]$. These dimensional changes have been attributed to the irradiation-induced ballistic displacement of carbon atoms which accumulate as interstitial clusters between the basal planes, forcing the planes apart [4-6]. However, there has been no experimental evidence for such interstitial clusters causing the dimensional change [7, 8]. Our recent in situ electron irradiation studies [9] showed the formation of edge dislocations via vacancy loops. The incomplete planes associated with these dislocations then grow via positive climb resulting in the formation of extra basal planes, which could be the dominant mechanism behind the irradiationinduced dimensional changes in graphite.

The response of bulk nuclear graphite to irradiation cannot be understood from the lattice response alone due to the complexity in the microstructure which is composed of various phases including a binder phase, randomly oriented filler particles, quinoline insoluble (QI) particles, macro- and micro-cracks (Mrozowki cracks) parallel to c-axis, etc. [2, 10-13]. At low doses of irradiation, the c-axis expansion is accommodated by the microcracks and the a-axis shrinkage results in net volume shrinkage. These microcracks are presumed to eventually close with further irradiation and the c-axis swelling is no longer accommodated resulting in net volume expansion of the graphite. This trend reversal in the dimensional change, known as "turnaround", is a function of the microstructure such as the degree of crystallite orientation, volume of the microcracks and gas evolution pores present in a given graphite grade. The lifetime of a graphite grade is defined as the dose at which the volume change returns to zero. These irradiation- 
induced microstructural changes have a profound impact on the physical properties such as the Young's modulus, fracture strength, and electrical and thermal conductivities [14-18]. The turnaround points for two isotropic graphite grades GraphNOL [14] and IG-110 [17] irradiated under similar conditions are $21 \mathrm{dpa}$ and $15 \mathrm{dpa}$, respectively, showing the effect of microstructure on the irradiation response. However, there is a lack of understanding of how the microstructure affects the irradiation response, as well as the physical properties, of these graphites in general.

We recently reported in detail the microstructural characteristics of various petroleum- and pitchbased nuclear graphites (IG-110, NBG-18 and PCEA) [13, 19] . The results showed a significant difference in the microstructure between the grades. For example, the pitch-based grade NBG-18 contains a higher concentration of binder phase and QI particles compared to the petroleumbased grades such as IG-110. In the present work, we report the effect of neutron irradiation on the microstructure of NBG-18 and IG-110 grade graphites. We report here for the first time the irradiation-induced response of the different microstructural features using transmission electron microscopy (TEM). We have also used high resolution transmission electron microscopy (HRTEM) to study the effect of neutron irradiation on the graphite lattice to understand the mechanism behind the irradiation-induced dimensional changes.

\section{Materials and Methods}

Nuclear grade graphite samples from several different manufactures were irradiated as a part of the first advanced graphite creep capsule (AGC-1) experiment at the Advanced Test Reactor National User Facility (ATR-NSUF). TEM specimens of IG-110 and NBG-18 grade graphites used in the present study were prepared from the cylindrical samples irradiated in a mechanically unstressed condition. The dimensions of the samples before irradiation were $12.5 \mathrm{~mm}$ in diameter and $6.4 \mathrm{~mm}$ in length. IG-110 (Toyo Tanso Co., Kagawa, Japan) is a petroleum-cokebased, fine-grained, iso-molded nuclear graphite and NBG-18 (SGL Group, Wiesbaden, Germany) is a pitch-coke-based medium-grained, vibration-molded nuclear graphite. The irradiation details of the samples used in the present study are provided in Table 1 and the complete details of AGC series of irradiation experiments can be found elsewhere [20].

TEM specimen-preparation steps and conditions used were exactly the same as those used for unirradiated samples [13]. Specimens were prepared by slicing the irradiated samples into $3 \mathrm{~mm}$ disks using a low-speed diamond saw. These disks were then mechanically thinned down to 20 
to $30 \mu \mathrm{m}$ in thickness. The final electron-transparency was achieved using a precision ion polishing system (PIPS 691; Gatan Inc., USA). Samples were typically milled using $5 \mathrm{keV}$ Ar ion beam for about $60-70 \mathrm{~min}$ at $5^{\circ}$ followed by a low angle polishing step at $2^{\circ}$ for $10 \mathrm{~min}$. Room-temperature TEM studies were performed at $200 \mathrm{kV}$ using a JEOL-2100 HR TEM (JEOL Ltd., Tokyo, Japan).

\section{Results and Discussion}

Figure 1 shows the bright-field TEM micrographs recorded from NBG-18 graphite samples irradiated to $1.54 \mathrm{dpa}$. Figure 1(a) shows the microstructure of a filler particle. NBG-18 is medium-grained nuclear graphite with an average filler size of about $1.6 \mathrm{~mm}$ and the TEM image shows one such particle. In general, filler regions can be identified by both their high crystallinity and the micro-cracks that are parallel to the basal planes. These micro-cracks are formed due to the thermal contraction-induced delamination of basal planes during the graphite manufacturing process. As seen in figure 1(a), the micro-cracks are tens of nanometers in width and hundreds of nanometers in length and exhibit a significant size distribution similar to nonirradiated samples [19]. The micro-cracks are also filled with amorphous carbon as seen clearly in figure 1(a) (indicated by arrows) which has only been discovered recently and has been shown not to be an artifact introduced by sample preparation [11]. As discussed above, these microcracks have a significant effect on the irradiation-induced dimensional changes. Figure 1(b) shows the micrograph recorded from a binder region embedded with rosette shaped QI particles. Coal-tar pitch is used as a binder in NBG-18 graphite, which naturally contains a high concentration of QI particles [13]. The solid QI particles are formed as a byproduct during the decomposition of coal or condensation of aromatic hydrocarbons on the walls of the cracking chamber during the petroleum refinement process. During graphite manufacture, these particles made of aromatic molecules graphitize to result in rosette shaped particles constituting a spiral of graphite strands. Unlike the filler particles, binder regions exhibit a variety of microstructural features such as randomly oriented fine graphite crystallites, microcracks, chaotic structures and QI particles.

Figure 2 shows the microstructural features of IG-110 graphite irradiated to 1.9 dpa. Figure 2(a) shows a filler particle with numerous micro-cracks of varying sizes. Figure 2(b) shows a binder region with chaotic structures and amorphous carbon with pores. Unlike in NBG-18, the 
concentration of QI particles in IG-110 is negligible due to the usage of petroleum-based pitch as the binder. In both NBG-18 and IG-110 (irradiated to 1.54 and 1.9 dpa, respectively), the QI particles are very similar in shape and size to those observed in the corresponding unirradiated graphite samples [13]. The microstructure does not show any evidence of irradiation-induced shrinkage of graphite along the c-axis or associated closing of micro-cracks. It is possible that the dimensional change is not significant enough to be seen under the TEM and is further complicated by the wide size distribution of these microcracks.

Figure 3(a-c) shows the bright-field micrographs recorded from the filler regions of NBG-18 graphite irradiated to 6.78 dpa. Figures 3(a) and (b) show filler particles with numerous microcracks similar to those present in unirradiated graphite. However, the cracks are fewer in number and there are some grains that show near complete absence of microcracks as shown in figure 3(c). The absence of microcracks in the filler particle shown in figure 3(c) indicates a substantial swelling of the graphite along the c-axis resulting in crack closing. The filler particles throughout the sample showed a varying degree of c-axis swelling and crack closing. The brightfield images of the filler particles that show crack closing also reveals the presence of significant diffraction contrast in the form of dark fringes (figure 3(c)) oriented perpendicular to the basal planes which could be indicative of intense structural damage in the form of bending and buckling of the basal planes. Figure 3(d) shows a SAED pattern recorded from the filler particle shown in figure 3(b) irradiated to $6.78 \mathrm{dpa}$. The presence of well-defined spots along the $\mathrm{c}^{*}$-axis indicates that the layered structure of the basal planes is still retained. It is also evident from the diffraction pattern that the micro-cracks are perpendicular to the c-axis and parallel to the basal planes. The arcing of diffraction spots is similar to non irradiated graphites which has been attributed to low angle twist boundaries which may be a result of basal plane delamination [13].

Figure 4 shows the microstructure of the filler particles in IG-110 graphite irradiated to 6.7 dpa. Figures 4(a) to (c) show three different filler particles with varying degrees of crack closing. Figure 4(a) shows a filler particle with most of the cracks closed due to irradiation induced swelling. Figures 4(b) and (c) show filler particles with some microcracks that are still open and it is interesting to note here that these unclosed microcracks are filled with amorphous carbon (indicated by arrows). It is possible that the presence of amorphous material could prevent these cracks from closing partially or completely. Like NBG-18, IG-110 also shows significant diffraction contrast as indicated by the numerous dark fringes oriented perpendicular to the basal 
planes, seen clearly in the magnified figure $4(d)$. The grains that show significant swelling and associated crack closure are the ones that show this kind of contrast, indicating a link between the two. It is not surprising that the cracks are not completely closed because the doses used in the present study ( $\approx 6.7 \mathrm{dpa})$ are much smaller than the typical dose at which "turnaround" occurs in these graphites (15 dpa in case of IG-110 [17] ).

The most striking effect of neutron irradiation was seen in the binder region of both the graphite grades. Figure 5 (a-c) shows the microstructure of the binder regions in NBG-18 graphite irradiated to $6.78 \mathrm{dpa}$. Figure 5 (a) shows a low magnification image of the binder region with numerous QI particles and figures 5(b) and (c) show magnified images of such QI particles from different regions of the binder. It can be seen clearly that the binder region looks highly densified with almost no residual micro-cracks. This type of densification of binder is seen throughout the sample. In the case of unirradiated graphites, the QI particles typically exhibit rosette shaped structures constituting a spiral of graphite strands [13]. Figures 5 (b) and (c) show the complete transformation of QI particles from such loosely packed rosettes to highly densified solid spheres. The structure of these spheres constitutes concentric strands of basal planes with the c-axis oriented along the radial direction of the sphere and the a-axis oriented along the circumference which is confirmed by the presence of concentric rings in the selected area electron diffraction (SAED) patterns (figure 5(d)) recorded from the QI particle shown in figure 5(c). The continuous variation in the orientation of the basal planes in the QI particles (equivalent to random orientation) results in the ring pattern. The non-uniform intensity distribution in the diffraction rings also indicate the presence of texture which could be because the QI particles are not perfectly spherical. The binder region of IG-110 irradiated to 6.7 dpa also showed a similar densification behavior as shown in figure 6(a). The transformation of QI particles from rosettes to solid spheres with onion-like structure is shown in figure 6(b). In the case of QI particles, irradiation-induced a-axis shrinkage should reduce the circumference of the graphitic strands which in turn should result in radial shrinkage of the particles. The graphitic strands should also increase in thickness due to c-axis swelling filling up the intra-particle voids. The combination of these two processes could be the mechanism behind the observed microstructural transformation of QI particles. A similar mechanism has been used to explain the irradiation-induced dimensional change behavior of graphite fiber composites with circumferential basal planes oriented parallel to the fiber axis [21]. The presence of cracks at the 
QI particle - binder matrix interface as seen in figures 5(b) and (c) (marked by arrows) indicates a mismatch in the shrinkage rates of the QI particles and the binder matrix. The micrographs from the binder regions in general show near complete densification without much residual open space. On the other hand, the filler particles still show the presence of numerous cracks. One explanation might be that the graphite particles in the binder are much smaller and randomly oriented, leading to a uniform swelling/densification in all directions. The dissimilarity in the densification behavior of various microstructural features elucidates the role of microstructure on the overall irradiation response and physical properties of nuclear graphites. As shown in Table 1, the macroscopic volume decreased for all the samples under study after irradiation due to the accommodation of c-axis swelling by the micro cracks. The net shrinkage in case of IG-110 irradiated to $6.7 \mathrm{dpa}$ is $4.3 \%$, where as it is $3.9 \%$ in case of NBG-18 irradiated to $6.78 \mathrm{dpa}$. NBG18 has a higher concentration of binder phase and QI particles compared to IG-110. It is possible that the filler particles contribute more to the decrease in volume by accommodating c-axis expansion via the closing of micro-cracks compared to the binder phase. Furthermore; shrinkage of QI particles creates new cracks around them reducing their contribution to macroscopic shrinkage.

The effect of irradiation on the graphite lattice and the mechanism responsible for irradiationinduced swelling has been further examined with the help of HRTEM. Figure 7(a) shows the lattice image recorded from a filler particle along a direction normal to [001] and depicts the basal planes of NBG-18 graphite irradiated to $6.78 \mathrm{dpa}$. The micrograph in figure 7(a) clearly shows the presence of breaking and bending of lattice planes caused by the formation of dislocations. Figure 7(b) shows a section of figure 7(a) magnified and noise-filtered. It shows the presence of extended interlayer defects connecting adjacent basal planes creating a pair of edge dislocations (marked by a solid circle) as well as prismatic dislocations (marked by solid rectangles). These findings are identical to those of the earlier work on in situ electron-beamirradiated graphites [9]. The theoretical calculations by Travethon et al. [22] showed that these extended defects connect the adjacent planes through continuous hexagonal sp2 bonding alone and can form via the aggregation of individual vacancy point defects. The presence of a few vacancy loops has also been noticed, but no interstitial loops. The room-temperature electronbeam-irradiation-induced swelling has been attributed mainly to the positive climb of prismatic dislocations resulting in the formation of new basal planes [9]. The same mechanism could be 
used to explain the swelling in neutron-irradiated graphite as well. In fact, the dislocation couple in the upper part of the figure 7(b) shows evidence for such dislocation climb. However, unlike the electron-irradiation case, most of the graphite lattice is still intact even after undergoing a damage of about $6.7 \mathrm{dpa}$ of neutron irradiation. The largely ordered structure is retained presumably because the neutron-irradiation was carried out at much higher temperatures (Table 1), giving enough thermal energy for the point defects to migrate and anneal out.

\section{Conclusions}

The effect of neutron irradiation on the microstructures of NBG-18 and IG-110 graphites has been studied using transmission electron microscopy. NBG-18 and IG-110 samples irradiated to 1.54 and $1.91 \mathrm{dpa}$, respectively, did not display any observable change in the microstructure. Samples of the same grades irradiated to 6.78 and 6.8 dpa, respectively, exhibited drastic changes in the microstructure. The filler particles showed significant closing of the microcracks indicative of c-axis swelling. The binder regions showed a complete densification with almost no microcracks and the QI particles exhibited a transformation from rosettes to solid spheres. The high resolution electron microscopic studies showed the presence of prismatic edge dislocations and evidence for the positive climb of these dislocations resulting in the formation of new basal planes which could be major contributing factor to the c-axis swelling.

\section{Acknowledgements}

This material is based upon work supported by the Department of Energy [National Nuclear Security Administration] under Award Numbers 00041394 / 00026 and DE-NE0000140. TEM studies were carried out at the Boise State Center for Materials Characterization (BSCMC. Furthermore, J. Kane acknowledges the funding of the Nuclear Regulatory Commission under the Nuclear Materials Fellowship Program (NRC-38-08-955). The authors are also thankful to Bryan Forsmann and Brian Jaques of Boise State University for their help with TEM sample preparation of irradiated graphites. 
References

[1] Allen TR, Sridharan K, Tan L, Windes WE, Cole JI, Crawford DC, et al. Materials challenges for Generation IV nuclear energy systems. Nucl Technol. 2008;162(3):342-57.

[2] Nightingale RE. Nuclear graphite. New York: Academic press 1962.

[3] Brocklehurst JE, Kelly BT. The dimensional changes of highly-oriented pyrolytic graphite irradiated with fast neutrons at $430^{\circ} \mathrm{C}$ and $600^{\circ} \mathrm{C}$. Carbon. 1993;31(1):179-83.

[4] Thrower PA, Reynolds WN. M icrostructural changes in neutron-irrdiated graphite. J Nucl Mater. 1963;6:221-6.

[5] Heerschap $M$, Schüller E. Vacancy and interstitial loops in graphite single crystals reactorirradiated at $900^{\circ}$ and $1200^{\circ} \mathrm{C}$. Carbon. 1969;7(5):624-5.

[6] Telling RH, Heggie MI. Radiation defects in graphite. Phil Mag. 2007;87:4797.

[7] Tanabe T, M uto S, Niwase K. On the mechanism of dimensional change of neutron irradiated graphite. Appl Phys Lett. 1992;61(14):1638.

[8] Muto S, Tanabe T. Damage process in electron-irradiated graphite studied by transmission electron microscopy. I. High-resolution observation of highly graphitized carbon fibre. Phil Mag A. 1997;76(3):679-90.

[9] Karthik C, Kane J, Butt DP, Windes WE, Ubic R. In-situ transmission electron microscopy of electron-beam induced damage process in nuclear grade graphite. J Nucl Mater. 2011;412(3):321-6.

[10] Jones AN, Hall GN, Joyce M, Hodgkins A, Wen K, Marrow TJ, et al. M icrostructural characterisation of nuclear grade graphite. J Nucl Mater. 2008;381(1-2):152-7.

[11] Wen KY, Marrow J, Marsden B. Microcracks in nuclear graphite and highly oriented pyrolytic graphite (HOPG). J Nucl Mater. 2008;381(1-2):199-203.

[12] Wen KY, Marrow TJ, Marsden BJ. The microstructure of nuclear graphite binders. Carbon. 2008;46(1):62-71.

[13] Karthik C, Kane J, Butt DP, Windes WE, Ubic R. M icrostructural characterization of next generation nuclear graphites. Microsc M icroanal. 2012;18(02):272-8.

[14] Burchell TD, Eatherly WP. The effects of radiation damage on the properties of GraphNOL N3M. J Nucl Mater. 1991;179-181, Part 1(0):205-8.

[15] Wu CH, Bonal JP, Thiele B. Thermal conductivity changes in graphites and carbon/ carbon fiber materials induced by low neutron damages. J Nuc M ater. 1994;212-215(Part 2):1168-73.

[16] Burchell TD, Snead LL. The effect of neutron irradiation damage on the properties of grade NBG10 graphite. J Nucl Mater. 2007;371(1-3):18.

[17] Ishiyama S, Burchell TD, Strizak JP, Eto M. The effect of high fluence neutron irradiation on the properties of a fine-grained isotropic nuclear graphite. Journal of Nuclear Materials.

1996;230(1):1-7.

[18] Kelly BT, Burchell TD. Structure-related property changes in polycrystalline graphite under neutron irradiation. Carbon. 1994;32(3):499-505.

[19] Kane J, Karthik C, Butt DP, Windes WE, Ubic R. M icrostructural characterization and pore structure analysis of nuclear graphite. J Nucl M ater. 2011;415(2):189-97.

[20] Grover SB. Status of the NGNP graphite creep experiments AGC-1 and AGC-2 irradiated in the advanced test reactor. Nucl Eng Design. 2014;271:275-82.

[21] Snead LL, Burchell TD, Katoh Y. Swelling of nuclear graphite and high quality carbon fiber composite under very high irradiation temperature. J Nucl Mater. 2008;381(1-2):55. 
[22] Trevethan T, Dyulgerova P, Latham CD, Heggie MI, Seabourne CR, Scott AJ, et al. Extended interplanar linking in graphite formed from vacancy aggregates. Phys Rev Lett. 2013;111(9):095501. 


\section{Figure Captions:}

Figure 1. Bright-field TEM micrographs of (a) filler region and (b) binder region in NBG-18 graphite irradiated to $1.5 \mathrm{dpa}$. Arrows in (a) mark the presence of amorphous carbon.

Figure 2. Bright-field TEM micrographs of (a) filler region and (b) binder region in IG-110 graphite irradiated to 1.9 dpa.

Figure 3. (a-c) Bright-field TEM micrographs of filler particles in NBG-18 graphite irradiated to $6.78 \mathrm{dpa}$ and (d) SAED pattern recorded from the particle shown in (c)

Figure 4. Bright-field TEM micrographs of filler particles in IG-110 graphite irradiated to 6.7 dpa. The arrows in (b) and (c) mark the presence of amorphous carbon.

Figure 5. (a-c) Bright-field TEM micrographs of the binder region with QI particles in NBG-18 graphite irradiated to $6.78 \mathrm{dpa}$ and (d) SAED pattern recorded from a QI particle shown in (c). Arrows in (b) and (c) mark the presence of cracks around the densified QI particles

Figure 6. Bright-field TEM micrographs of the binder region with QI particles in IG-110 graphite irradiated to $6.7 \mathrm{dpa}$.

Figure 7. (a) High resolution TEM image of a filler particle in NBG-18 graphite irradiated to 6.8 dpa showing the presence of defects in the lattice. (b) is a noise filtered image of a section of (a) showing the presence of dislocations. Distance between two black fringes corresponds to (002) inter-planar spacing $(\approx 0.37 \mathrm{~nm})$. 

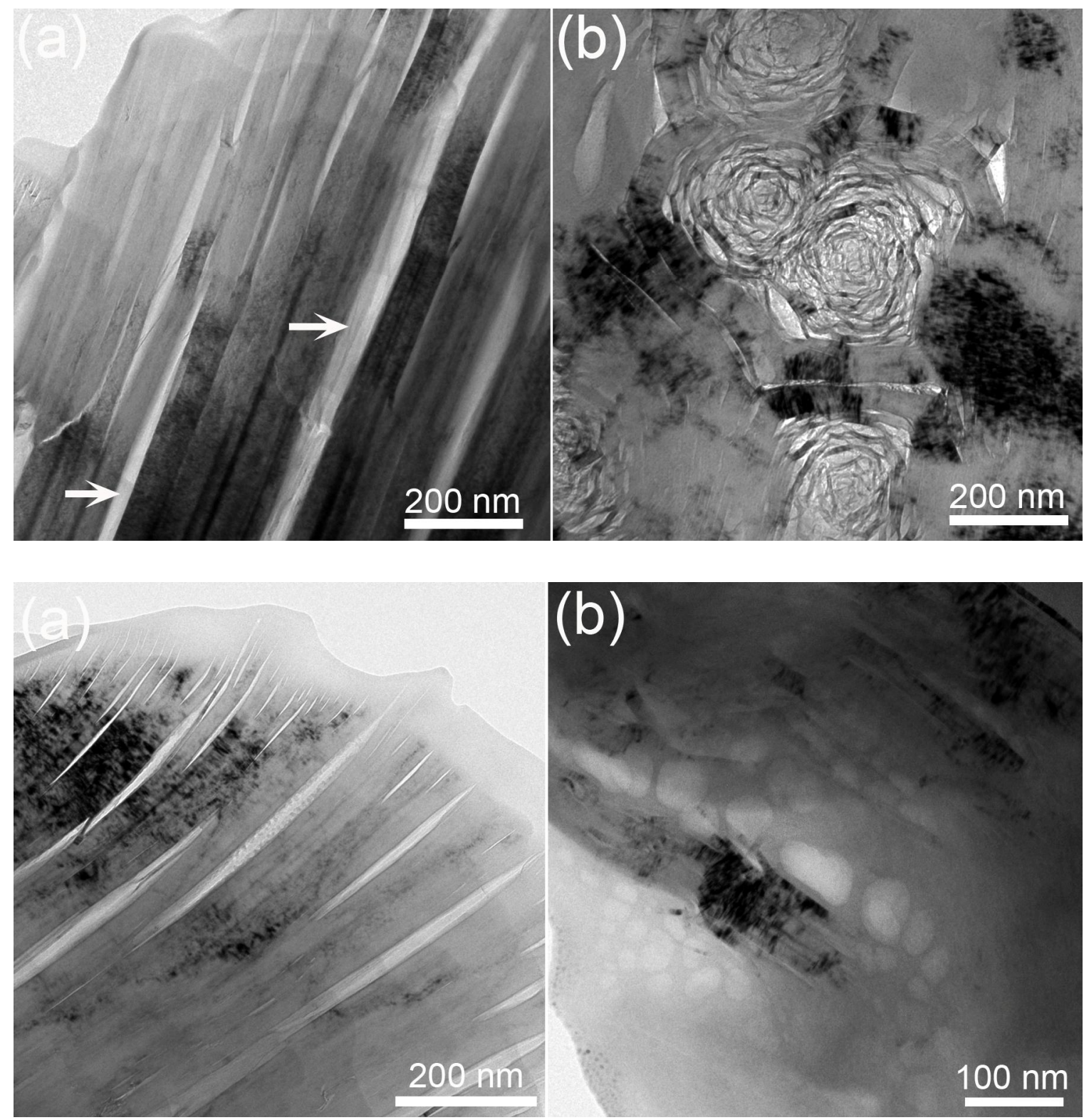

(b)

$100 \mathrm{~nm}$ 


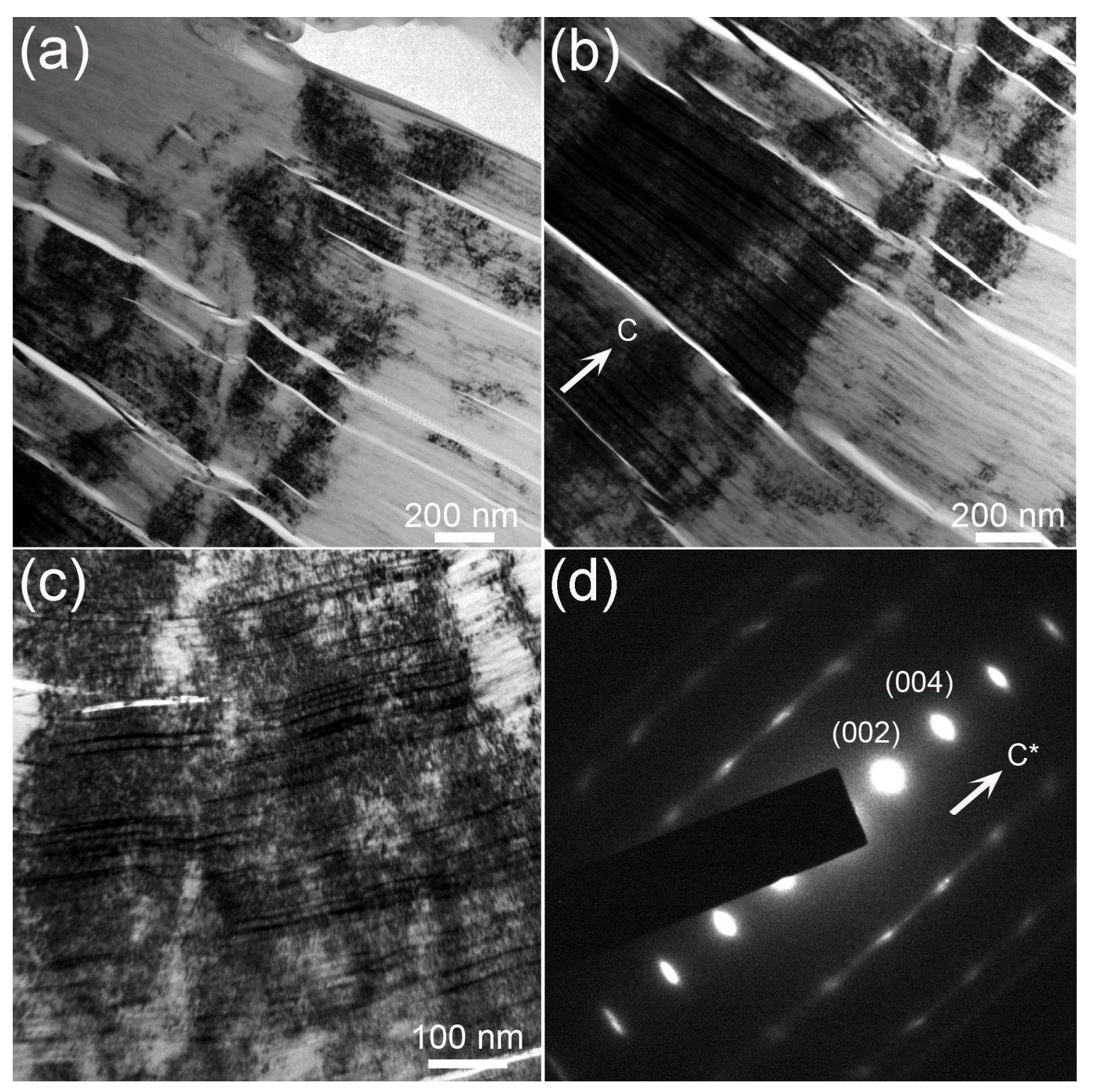




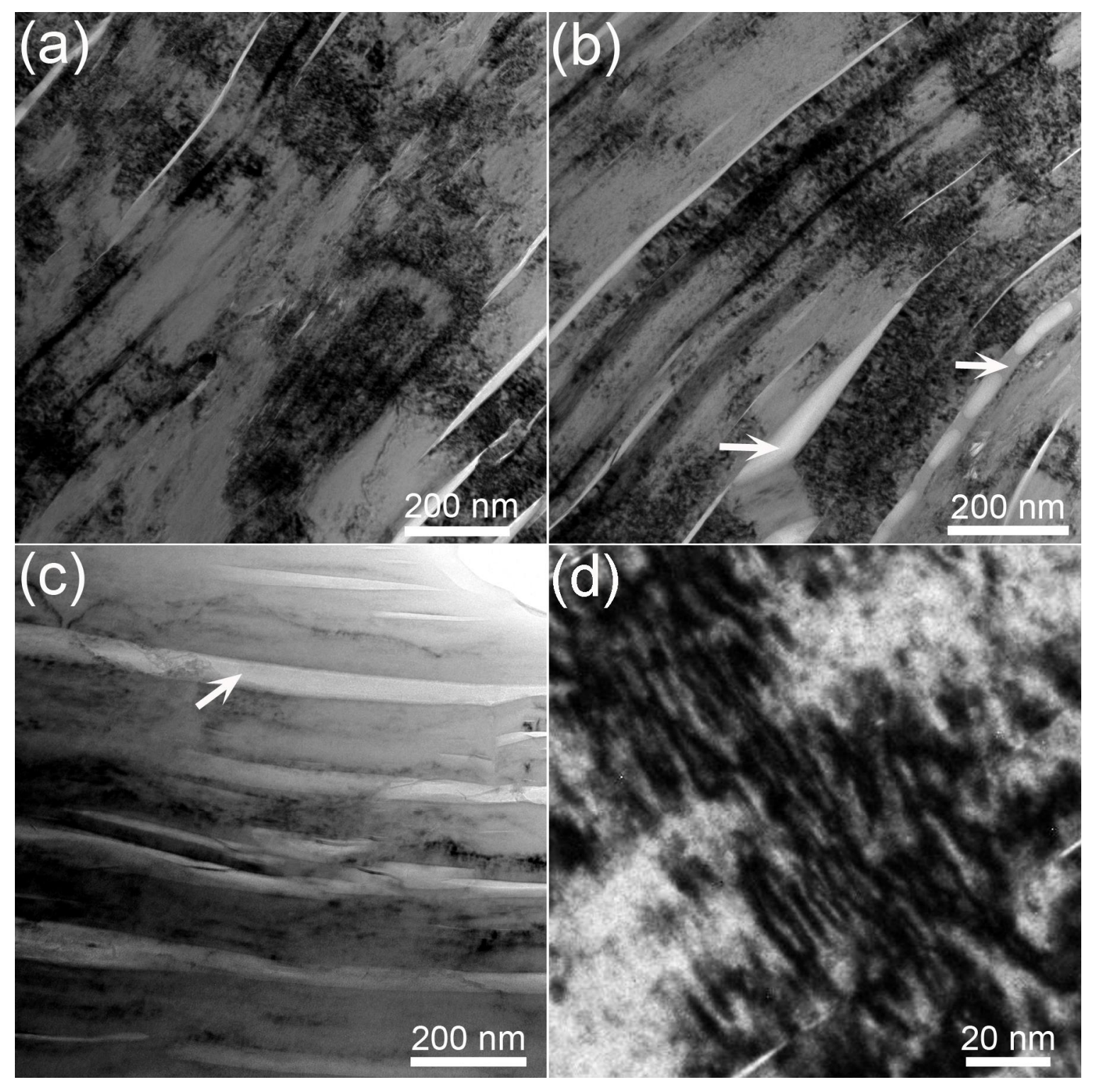




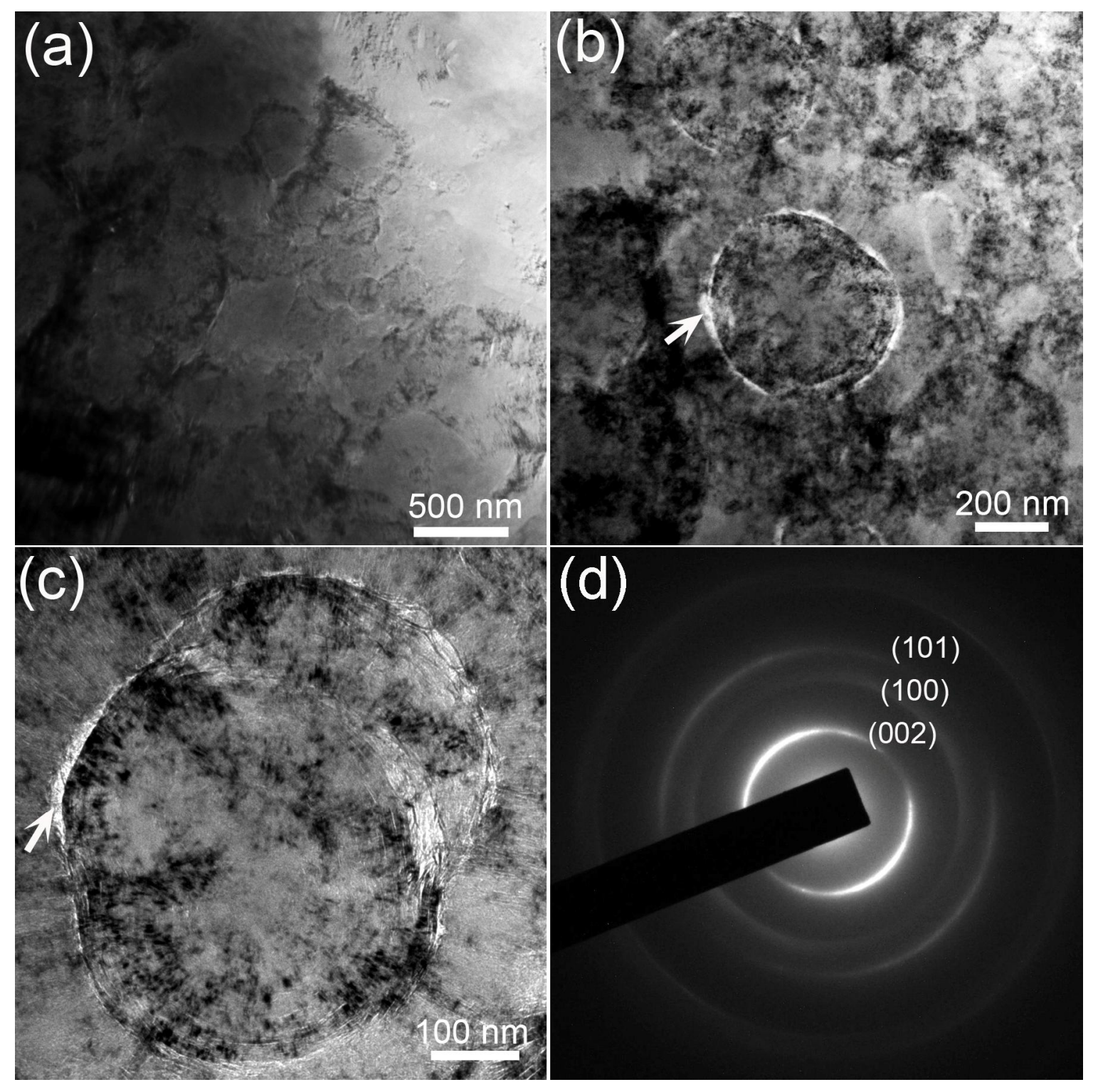




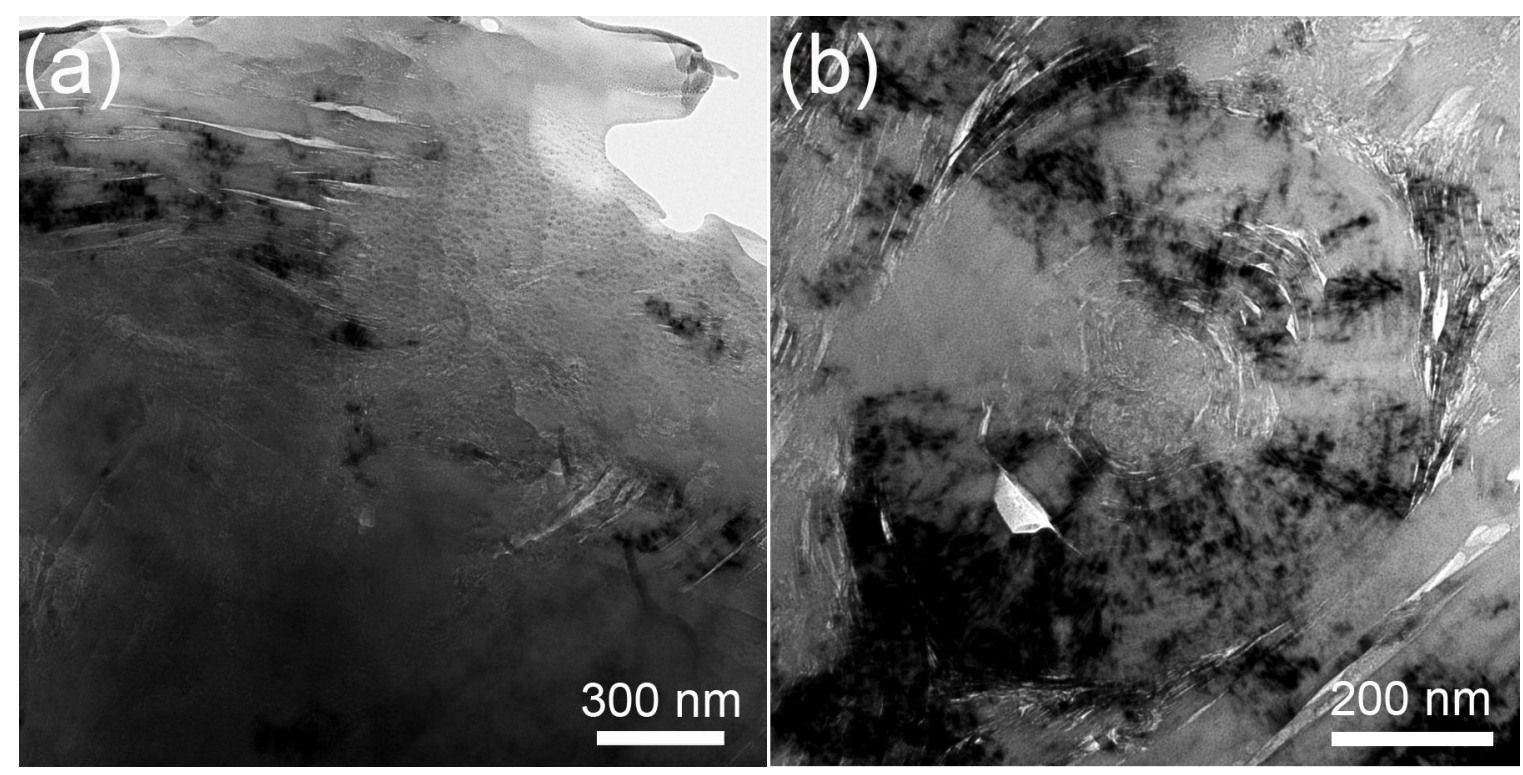



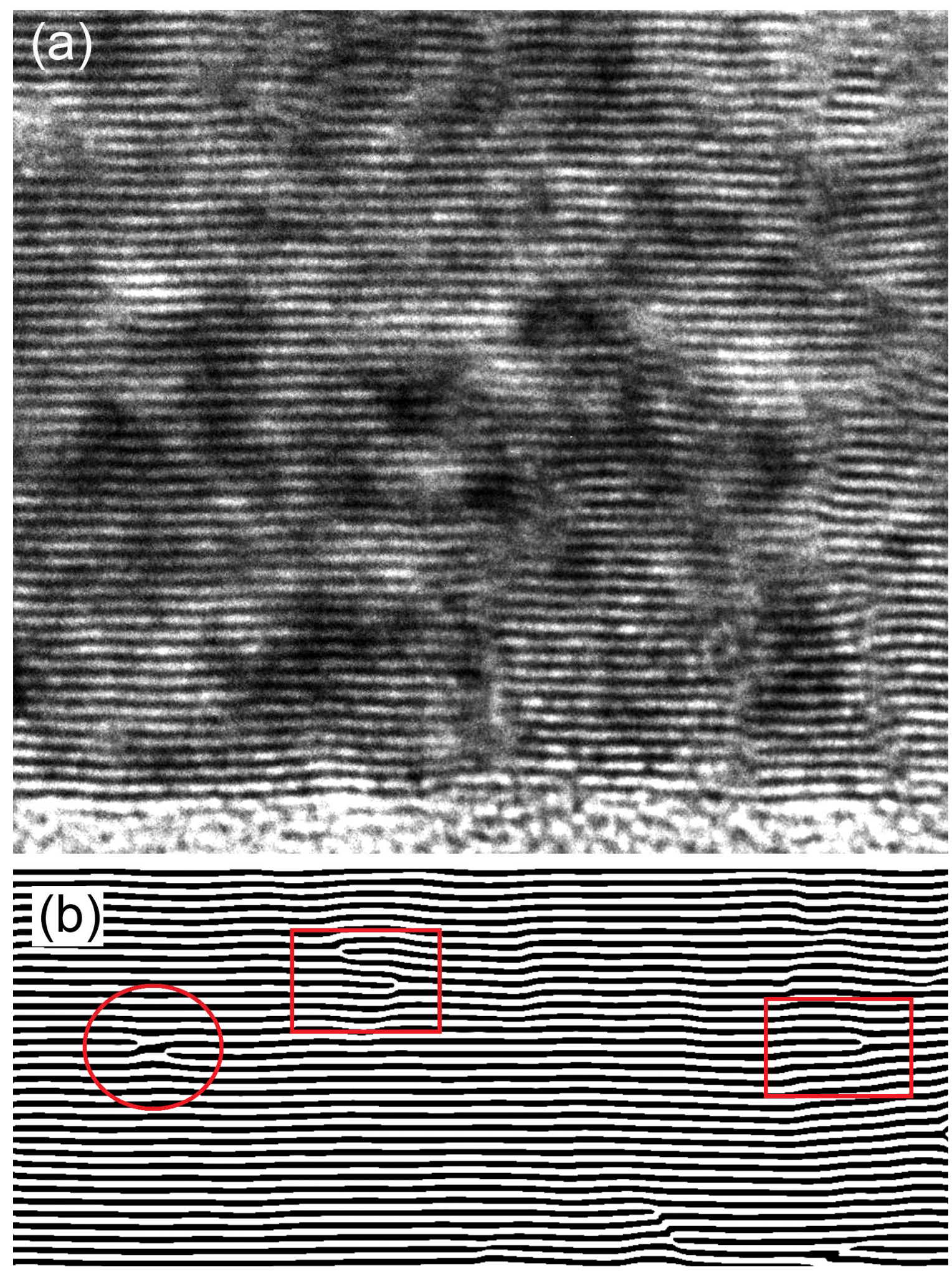
Table 1. Irradiation conditions used for various graphite grades and the net irradiation induced volume change

\begin{tabular}{|c|c|c|c|}
\hline Grade & Dose (dpa) & $\begin{array}{c}\text { Temperature } \\
\left({ }^{\circ} \mathrm{C}\right)\end{array}$ & $\begin{array}{c}\text { Volume change } \\
(\%)\end{array}$ \\
\hline NBG-18 & 1.54 & 430 & -0.6 \\
NBG-18 & 6.78 & 678 & -3.9 \\
IG-110 & 1.91 & 451 & -1.1 \\
IG-110 & 6.70 & 674 & -4.3 \\
\hline
\end{tabular}

